\title{
Influencia Del Hábito De La Lectura En La Enseñanza Aprendizaje Del Vocabulario En Estudiantes De Octavo Año De Educación Básica
}

\author{
Cando Carrillo María Liliana \\ Chuquimarca Once Doris Alexandra
}

Licenciada en Ciencias de la Educación Profesora de Idiomas inglés

Docente de la Escuela Superior Politécnica de Chimborazo,

Centro de Idiomas, Ecuador

Pazmiño Pavón Luis Even

Magister en lingüística aplicada al aprendizaje del inglés

Docente de la Escuela Superior Politécnica de Chimborazo,

Centro de Idiomas, Ecuador

Doi:10.19044/esj.2018.v14n22p187 URL:http://dx.doi.org/10.19044/esj.2018.v14n22p187

\begin{abstract}
The objective of the research was to determine the reasons why eighth year parallel "A" students of Primary Education at the "Maldonado High School" do not improve their vocabulary during the learning process through reading. To do so, the types of reading that the teacher uses in classes were diagnosed; we identified the techniques used to teach vocabulary and finally proceeded with the inference of reasons because students lack vocabulary when they read. The research was diagnostic and the ethnographic method was used to describe and analyze the sociocultural environment of the research context. The sample was intentional and represent twenty-eight students and one teacher. The data were obtained by means of participatory observation with an observation list, applied in May and June of 2016. The research was inductive and field, as general conclusions were obtained from observation, classification and study of facts, based on the direct collection of information from the reality in which it was investigated. The results obtained were tabulated, analyzed and interpreted and determined that students have a bad habit and do not follow a technical reading process.
\end{abstract}

Keywords: Reading Habit, Influence, Teaching Learning, Vocabulary.

\section{Resumen}

El objetivo de la investigación fue determinar las razones por las cuales los estudiantes de octavo año de educación básica octavo año paralelo "A" de 
educación básica de la Unidad Educativa "Maldonado", no mejoran su vocabulario durante en el proceso de aprendizaje mediante la lectura. Para ello, se indagó los tipos de lectura que el profesor usa en clases; se identificó las técnicas usadas para enseñar vocabulario y finalmente se procedió con la inferencia de las razones porque los estudiantes carecen de vocabulario cuando leen. La investigación fue diagnóstica y el método etnográfico se utilizó para describir y analizar el entorno sociocultural del contexto de la investigación. La muestra fue intencionada y representan 28 estudiantes y 1 profesora. Los datos se obtuvieron mediante la observación participativa con una lista de observación, aplicada en los meses de mayo y junio de 2016. La investigación fue inductiva y de campo, ya que se obtuvo conclusiones generales de la observación, clasificación y estudio de hechos, basándose en la recopilación directa de información de la realidad en que se investigó. Los resultados obtenidos fueron tabulados, analizados e interpretados y determinaron que los estudiantes tienen un mal hábito y que no siguen un proceso técnico de lectura.

Palabras claves: Hábito De Lectura, Influencia, Enseñanza Aprendizaje, Vocabulario

\section{INTRODUCCIÓN}

Según Goodmann \& Goodmann (1998), para el modelo interactivo de lectura de investigadores, "se obtiene lectura del significado del texto escrito y por lo tanto el lector debe procesar, como lenguaje, información visual ofrecida por el texto; además, la lectura es un proceso psicolingüístico de creación y confirmación de hipótesis del conocimiento previo del lenguaje y el mundo, un fenómeno exclusivamente perceptivo".

Esto significa que el proceso de lectura no es simple, pero implica mucho más que eso; en la medida en que, al leer no solo se puede percibir lo que se escribe; sino también, los sentimientos que el autor quería expresar cuando escribió su composición.

Goodmann menciona en su declaración, que a partir del conocimiento previo sobre un tema, es posible crear y confirmar una hipótesis. Cuanto más se lee se obtiene conocimiento previo y mejor comprensión de lectura. Lo que significa que el proceso de lectura no es simple, e implica que lo que se lee no solo se puede percibir, sino también se debe entender los sentimientos que el autor expresa cuando escribe su composición.

Menciona en su declaración que a partir del conocimiento previo sobre un tema, es posible crear y confirmar una hipótesis, estar de acuerdo con su pensamiento porque cuanto más se lee y se obtiene conocimiento previo, mejor comprensión de lectura. "No hay sentido sin leer". Pero por otro lado, se creía que la comprensión no se puede lograr sin usar el significado para decodificar el texto. 
El significado que el lector asigna al texto dependerá de factores, como el propósito del lector, su cultura social, sus antecedentes, su control lingüístico, sus actitudes y 5 esquemas conceptuales. Esto sugiere que los procesos de escritura y lectura son dinámicos y constructivo.

La lectura es la capacidad de entender las palabras contenidas en un documento y hacer uso del conocimiento para el crecimiento y el desarrollo personal. Esto implica obtener el significado de la información registrada impresa o no impresa en la vida de un individuo. Las personas leen por diferentes razones y propósitos, algunos de los cuales incluyen para el placer, el ocio, la relajación, la información y para el conocimiento.

Nuttall (1982), "La lectura de es una parte esencial de la instrucción lingüística en todos los niveles porque apoya el aprendizaje de múltiples maneras", especialmente cuando los estudiantes están aprendiendo un nuevo idioma. Los docentes deben proporcionar una variedad de textos tales como; revistas, historias de amor, novelas, cuentos, periódicos, etc. Los estudiantes son capaces de elegir la mejor opción para ellos y aprenden vocabulario nuevo y transmiten el significado del texto.

Por otro lado, la motivación es el factor más importante para el éxito en la inclinación del lenguaje. Los maestros, deben motivar a sus estudiantes en cada clase porque los estudiantes tienen problemas en casa, por eso no se concentran en las clases de inglés.

Según Torres (2010), entre las estrategias para motivar a los estudiantes, tenemos la de introducir variedad; donde la profesora debe dejar que los estudiantes elijan el tipo de lectura que les gusta, pues debe usar nuevos métodos para enseñar tales como: debates, discusiones, dramatizaciones, lluvia de ideas.

También se menciona el mejorar la atmósfera del aula de inglés; donde los estudiantes deben tener confianza en sí mismos, siendo capaces de asumir riesgos. El profesor mejora la atmósfera del aula con afiches, cuadros, títeres, etc. Es una buena manera para que los estudiantes no tengan miedo de participar en la clase y se sientan más cómodos. Por otro lado se puede utilizar material didáctico estimulante, que les gusta a los estudiantes aprender, a través de materiales didácticos relacionados con el tema; eso ayuda a hacer la clase más interesante y que los estudiantes no se aburran. Y finalmente hacer que los estudiantes utilicen el inglés en clase. Para ello, la profesora debe estar seguro de que sus estudiantes están aprendiendo a leer en inglés en cada clase y en el hogar.

Se recomienda que se recompense el rendimiento del alumno, proporcionándole atención, mostrando entusiasmo, energía y ayudándole a reconocer la importancia de la lectura. 
Los estudiantes deben leer a menudo porque cuando leen, aprenden cosas nuevas y enriquecen su vocabulario, e induce a sus cercanos al hábito de leer.

Los profesores tienen un gran desafío con sus estudiantes, ya que deben usar diferentes técnicas para motivarlos. Cuando los estudiantes están motivados en clase, se sienten seguros de sí mismos y demuestran sus habilidades y conocimientos recién adquiridos en el aula. Les ayuda a ganar confianza permanecer seguros de sí mismo.

Según Beale (2014), sustenta que existen estrategias para la lectura entre ellas: el escaneo, que es un tipo de lectura donde se obtienen las ideas principales del libro y es usado para leer más en menos tiempo; además existe el escaneo que se utiliza para acelerar la lectura y se utiliza para la exploración, donde se analiza la información específica e importante. El escaneo permite a los estudiantes encontrar detalles y otra información de forma apresurada.

Según Wilkins (1972), sustenta que "Aunque sin gramática muy poco puede ser transmitido, sin vocabulario nada puede ser transmitido". El vocabulario es esencial para la comunicación, si bien es cierto, el aprendizaje de vocabulario no lo es todo, pero ayuda a tener más fluidez y mejorar el proceso de comunicación.

Lo que implica conocer la forma y su significado, pues al conocer el significado de una palabra no es sólo saber su significado de diccionario, también significa conocer las palabras comúnmente asociadas con él (sus colocaciones), así como su connotación, incluyendo el registro y sus acrecentamientos culturales.

El vocabulario es tener un amplio conocimiento de las palabras, que conducen a tener una expresión correcta durante la comunicación. La adquisición del vocabulario comienza a una edad temprana, tal como el niño comienza a asociar términos con imágenes. Él asocia la palabra mamá con la mujer que se preocupa por él todo el tiempo.

Según Bamberger (1975), se puede crear hábitos de lectura considerando la promoción por parte de los padres, explorando posibilidades de ascenso en la enseñanza preescolar y jardín de infantes y algo importante que es la influencia de la profesora.

Los maestros al hacer pequeñas "dosis de hábito" en las actividades diarias de ocio y como tarea, de forma sistemáticamente a lo largo de la carrera escolar del niño -sin forzar, pero naturalmente-; permitirán que se acostumbren a generar un hábito de lectura y no se someterán al factor de cansancio y superarán el trabajar con lecturas largas como por ejemplo los libros.

El trabajo de la profesora no se limita a despertar la convicción y el entusiasmo por la importancia de los libros: debe estar en condiciones de practicar un buen volumen lecturas acordes para niños con libros específicos, 
con el fin de que los niños durante el proceso perciben una discusión de carácter participativo en el aula, que fortalecerá su confianza y motivará la lectura

En Ecuador, el idioma inglés no se ha considerado una prioridad porque es un país de habla hispana, pero con el avance del mundo tecnológico y el alcance del desarrollo global, es fundamental conocer el idioma inglés.

El contexto de la investigación fue formulado porque cuando se desarrollaron las prácticas pre profesionales, se observó que los estudiantes tenían dificultades para aprender vocabulario cuando estaban leyendo. Es por eso que se lo analizó en base a su contexto y considerando la influencia del hábito de la lectura en la enseñanza práctica del vocabulario a los estudiantes de octavo año "A" de Educación Básica de la Unidad Educativa "Maldonado", en la ciudad de Riobamba, durante el año académico 2015- 2016"

Aunque los estudiantes en el octavo año "A" reciben un total de 5 horas a la semana divididas en 1 hora diaria y tienen el libro del estudiante de inglés y el libro de trabajo Nivel 1 de (Jorge Enrique Muñoz Oyola) del Ministerio de Educación. Se cree que el problema surge debido a los malos hábitos de lectura en los estudiantes y maestros, como se vio durante el desarrollo de las prácticas. Se observó que 2 durante su tiempo libre los estudiantes no toman un libro, revista, periódico o novela para leer.

\section{METODOLOGÍA}

La investigación tiene enfoque cualitativo, ya que se basa en el uso de descripciones de rendimiento para analizar el comportamiento humano. El estudio se llevó a cabo mediante las técnicas: observaciones directas y entrevistas estructuradas. El método etnográfico se utilizó para describir y analizar el entorno sociocultural del contexto de la investigación, para recolectar datos a través de la observación participativa.

La investigación fue inductiva y de campo, ya que se obtuvo conclusiones generales de observación, clasificación y estudio de hechos. La investigación se basó en una recopilación directa de información de la realidad en que se investigó. Además, la investigación fue documental ya que se recompiló y analizó la información, tanto bibliográfica como académica.

La muestra fue intencionada, que estuvo integrada por 28 estudiantes y una profesora de octavo, del paralelo "A" en Unidad Educativa "Maldonado". La muestra fue escogida, basándose en experiencias previas como pasantías pre profesionales cuando observó el número excesivo de estudiantes, la mayoría de ellos tienen un estatus económico medio, que provienen de zonas rurales y muchos estudiantes no tienen una estabilidad familiar. Sin embargo, la mayoría de ellos muestran interés en la clase de inglés. 
Para recopilar la información, se realizaron los procedimientos legales en la unidad educativa y se designó la muestra antes mencionada para ser intervenida. La conexión entre la profesora y los estudiantes fue positiva y permitió a obtener los datos de forma objetiva, la misma que fue activa y los resultados finales fueron puestos en consideración con el profesor de la clase.

El instrumento de recolección fue una lista de cotejo para evaluar a los estudiantes; mientras que con el profesor se aplicó una entrevistas guiada compuesta de un conjunto de preguntas, que fueron elaboradas en base los hechos y aspectos interesados en la investigación.

Los aspectos observados en la investigación, ayudaron a determinar las razones por las que los estudiantes no mejoran su aprendizaje del vocabulario en el hábito de la lectura. Para ello, se realizó un estudio descriptivo a través de tablas estadísticas que muestran la información obtenida y que permite el análisis e interpretación de la misma.

El esquema que se desarrolló en la investigación, inició descubriendo los tipos de lectura que el profesor usa en clases para enseñar vocabulario. Luego se identificó las técnicas usadas por la profesora para enseñar vocabulario cuando leen. Finalmente se procedió con la inferencia de las razones porque los estudiantes de octavo año "A" de educación básica de la Unidad Educativa "Maldonado" carecen de vocabulario cuando leen.

La revisión de la información sobre las variable intervinientes, permitieron aplicar la guía de observación, de lo cual se clasificó el 100\% de los datos recolectados según los parámetros propuestos utilizados durante un mes para categorizar e identificar los datos. Una vez identificados los datos se procedió con el análisis e interpretación de resultados que están descritos en los resultados y las conclusiones.

\section{RESULTADOS}

Análisis de la hoja de observación aplicada a la clase con los estudiantes del octavo año de Educación Básica "A" y docente de la Unidad Educativa "Maldonado".

1. Descubrimiento de los tipos de lecturas que el profesor utiliza en clase para enseñar el vocabulario.

Pregunta 1. ¿Los estudiantes escanean la lectura (para obtener detalles).?

Tabla 1. Escaneo de lectura

\begin{tabular}{cc}
\hline & Frecuencias \\
\hline SI & 10 \\
No & 18 \\
\hline
\end{tabular}

Fuente: Encuesta

Elaborado por: Cando Liliana, 2016 


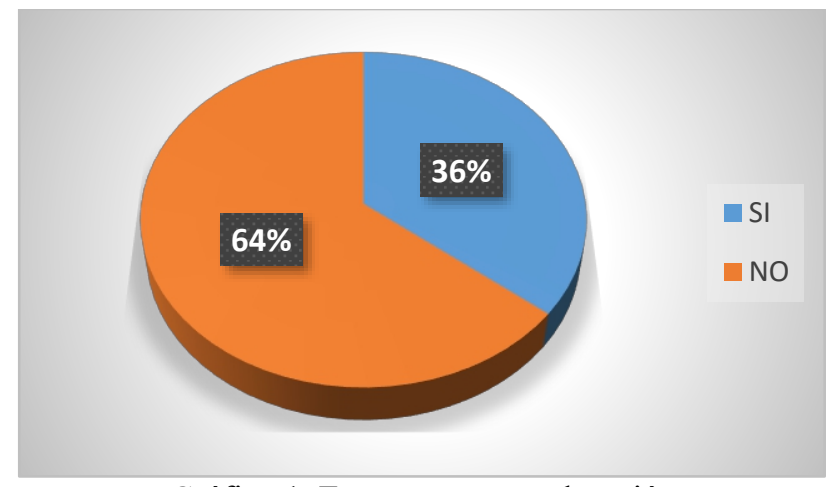

Gráfico 1. Escaneo y no exploración

Pregunta 2. ¿El profesor utiliza las técnicas de lectura extensiva e intensiva con los estudiantes?

Tabla 2. Técnica de lectura extensiva e intensiva aplicada por los estudiantes.

\begin{tabular}{cc}
\hline \multicolumn{2}{c}{ Frecuencias } \\
\hline SI & 28 \\
NO & 0 \\
\hline \multicolumn{3}{c}{ Fuente: Encuesta } \\
Elaborado por: Cando Liliana, 2016
\end{tabular}

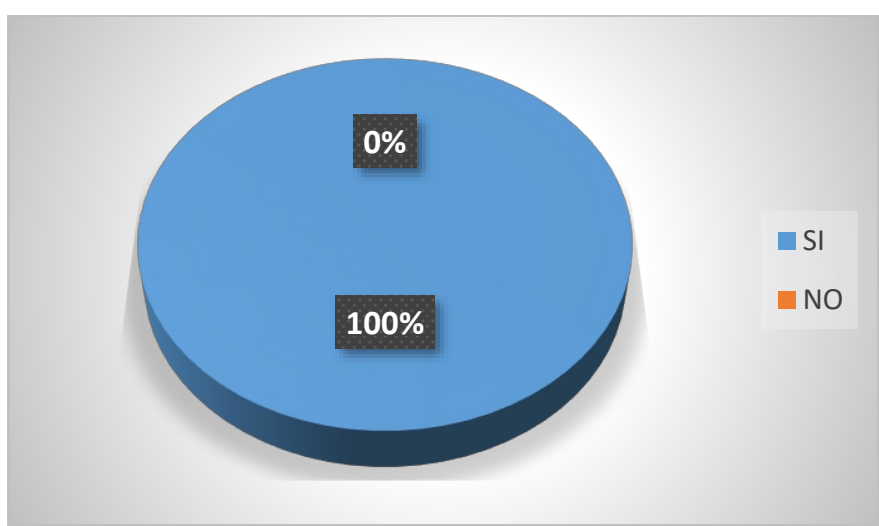

Gráfico 2. Técnica de lectura extensiva e intensiva aplicada por los estudiantes.

\section{Deducción de las razones por las que los estudiantes, carecen de vocabulario al leer.}

Pregunta 3. ¿Los estudiantes tratan de obtener el significado de palabras desconocidas por sí mismos?

Tabla 3. Obtención del significado de palabras de inglés a español

\begin{tabular}{cc} 
& Frecuencias \\
\hline $\mathrm{Si}$ & 8 \\
$\mathrm{No}$ & 20 \\
\hline \multicolumn{3}{c}{ Flaborado por: Cando Liliana, 2016}
\end{tabular}




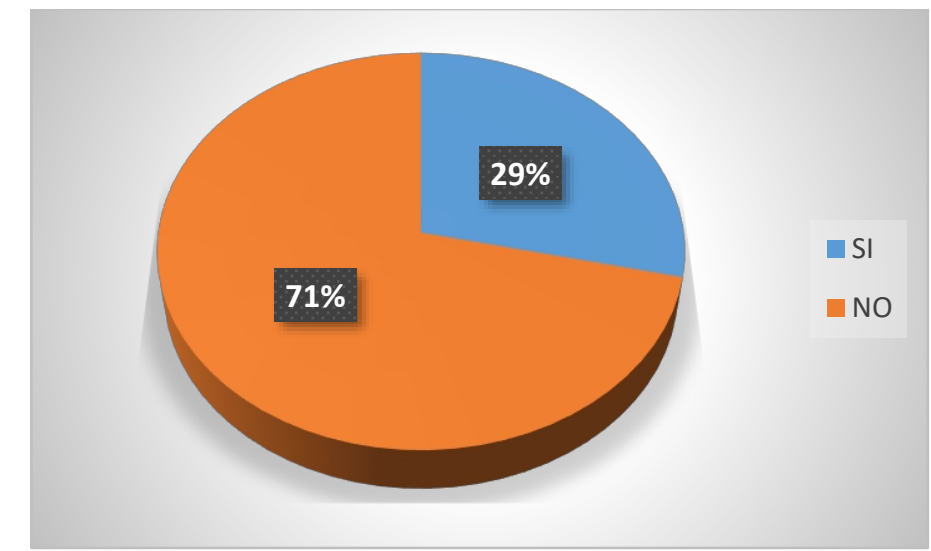

Gráfico 3. Obtención del significado de palabras de inglés a español

Pregunta 4 ¿Los estudiantes preguntan al profesora por el significado de alguna palabra en inglés?

Tabla 4. Preguntas del significado de palabras de inglés a español

\begin{tabular}{cc} 
& Frecuencia \\
\hline $\mathrm{Si}$ & 2 \\
$\mathrm{No}$ & 26 \\
\hline
\end{tabular}

Fuente: Encuesta

Elaborado por: Cando Liliana, 2016

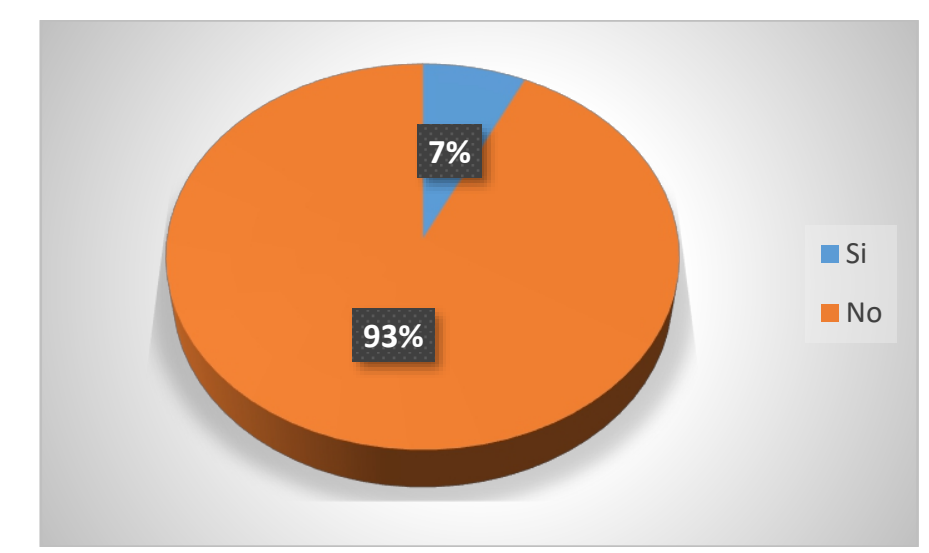

Gráfico 4. Obtención del significado de palabras de inglés a español

Pregunta 5. ¿Los estudiantes usan palabras nuevas en situaciones de la vida real?.

Tabla 5. Estudiantes que usan palabras nuevas en situaciones de la vida real

\begin{tabular}{cc}
\hline & Frecuencia \\
\hline $\mathrm{Si}$ & 0 \\
$\mathrm{No}$ & 28 \\
\hline
\end{tabular}

Fuente: Encuesta

Elaborado por: Cando Liliana, 2016 


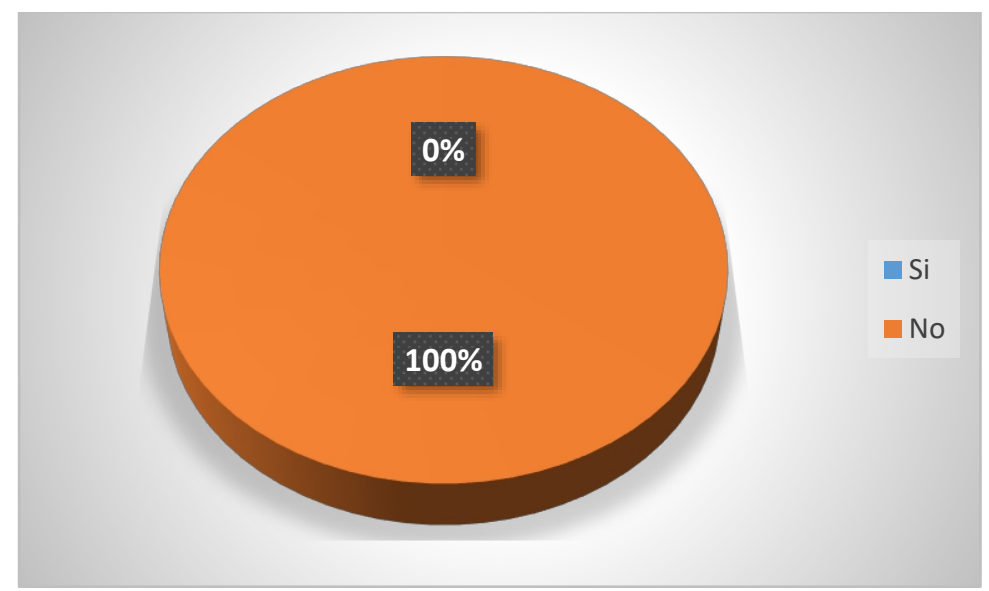

Gráfico 5. Estudiantes que usan palabras nuevas en situaciones de la vida real

Pregunta 6. ¿El docente practica el hábito de leer con los estudiantes?.

Tabla 6. Práctica el hábito de leer con los estudiantes

\begin{tabular}{cc}
\hline & Frecuencia \\
\hline $\mathrm{Si}$ & 10 \\
$\mathrm{No}$ & 18 \\
\hline
\end{tabular}

Fuente: Encuesta

Elaborado por: Cando Liliana, 2016

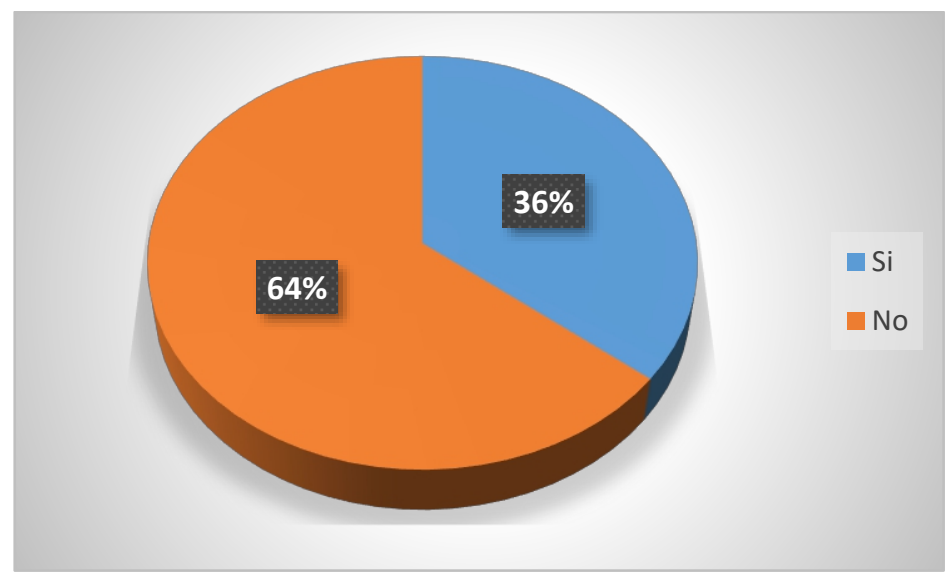

Gráfico 6. Práctica el hábito de leer con los estudiantes

Pregunta 7. ¿Los estudiantes son alentados por el docente a leer?.

Tabla 7. Estudiantes alentados a leer por el docente.

\begin{tabular}{cc}
\hline & Frecuencia \\
\hline $\mathrm{Si}$ & 0 \\
$\mathrm{No}$ & 28 \\
\hline
\end{tabular}

Fuente: Encuesta

Elaborado por: Cando Liliana, 2016 


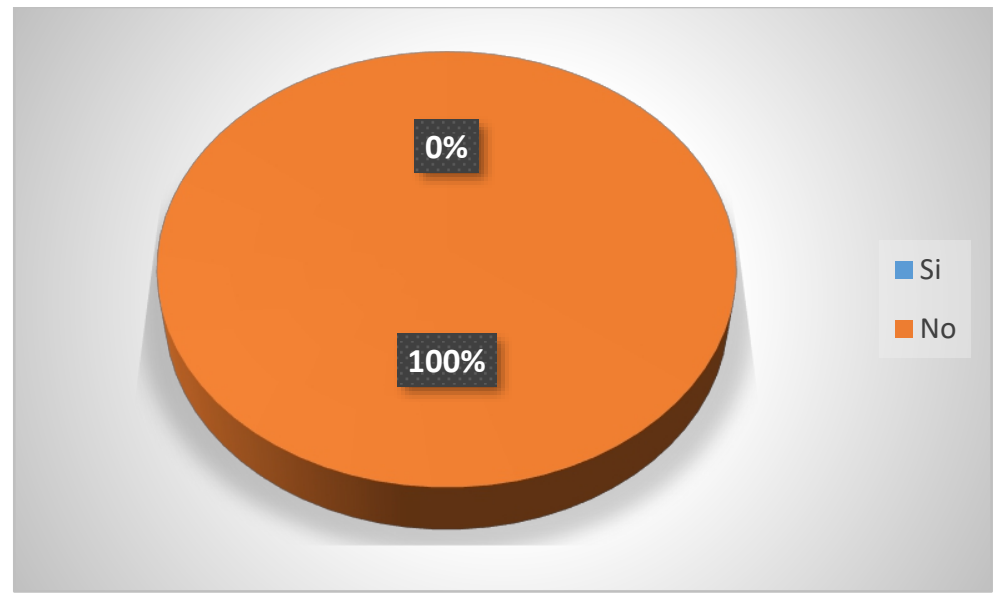

Gráfico 7. Estudiantes alentados a leer por el docente.

Pregunta 8. ¿Los estudiantes entienden el texto?

Tabla 8. Estudiantes que entienden el texto

\begin{tabular}{cc}
\hline & Frecuencia \\
\hline $\mathrm{Si}$ & 6 \\
$\mathrm{No}$ & 22 \\
\hline
\end{tabular}

Fuente: Encuesta

Elaborado por: Cando Liliana, 2016

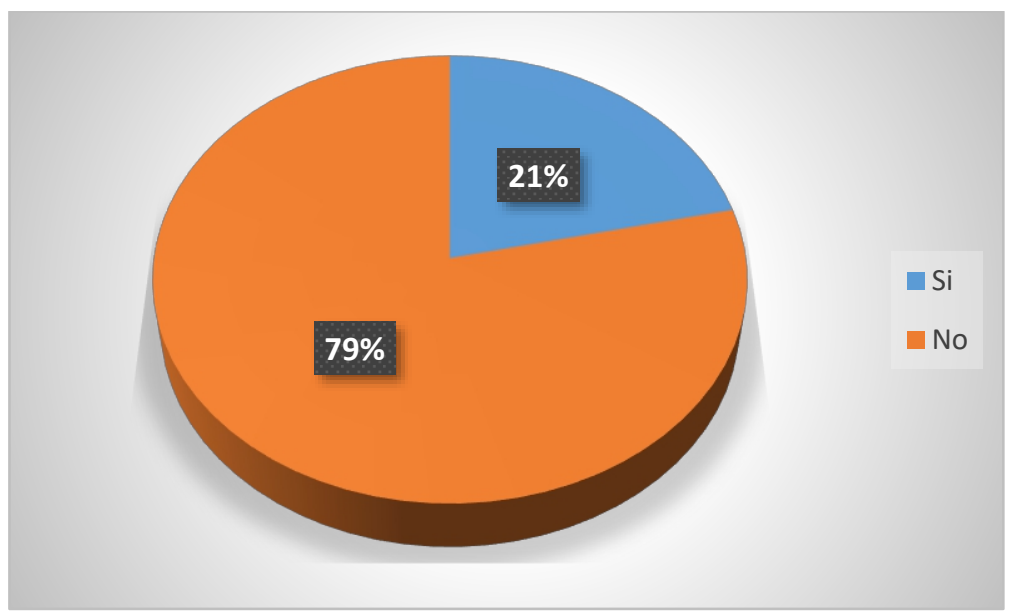

Gráfico 8. Estudiantes que entienden el texto

Pregunta 9. ¿Los estudiantes subrayan las palabras desconocidas?.

Tabla 9. Estudiantes que subrayan las palabras desconocidas.

Frecuencia

\begin{tabular}{cc}
\hline Si & 10 \\
No & 18 \\
\hline
\end{tabular}

Fuente: Encuesta

Elaborado por: Cando Liliana, 2016 


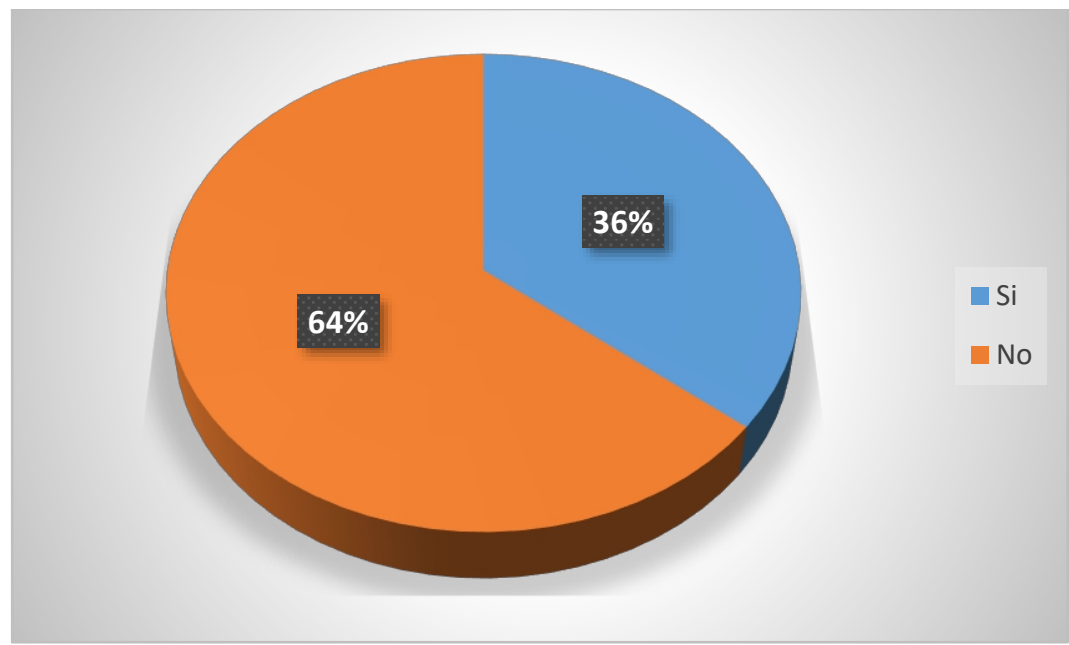

Gráfico 9. Estudiantes que subrayan las palabras desconocidas.

En la siguiente tabla se considera un resumen del procesamiento y análisis de la aplicación de los instrumentos aplicados a la profesora como a los estudiantes por lo que se considera la siguientes siglas en los temas: $(\mathrm{P})$ Profesor, (E) estudiantes.

Tabla 10: Procesamiento de datos, análisis

\begin{tabular}{|c|c|c|}
\hline OBJETIVOS & TEMAS & ANÁLISIS \\
\hline \multirow{4}{*}{$\begin{array}{l}\text { 1.- Descubrir los tipos de } \\
\text { lecturas que el profesor utiliza } \\
\text { en clase para enseñar } \\
\text { vocabulario a los estudiantes de } \\
\text { octavo año "A" de educación } \\
\text { básica de la unidad educativa } \\
\text { "Maldonado". }\end{array}$} & $\begin{array}{l}\text { (P) Los estudiantes conocen y aplican } \\
\text { diferentes tipos de lectura en la clase. }\end{array}$ & $\begin{array}{l}\text { Durante las observaciones realizadas, el } \\
\text { profesor aplicó la exploración como el tipo } \\
\text { principal de lectura con sus estudiantes. }\end{array}$ \\
\hline & $\begin{array}{l}\text { (P) Los estudiantes hojean la lectura } \\
\text { (para obtener la idea general). }\end{array}$ & $\begin{array}{l}\text { Los estudiantes aplicaron sólo lectura rápida } \\
\text { y obtuvieron las ideas generales del texto y } \\
\text { por lo tanto no dar respuestas adecuadas a las } \\
\text { preguntas formuladas por la profesora. }\end{array}$ \\
\hline & $\begin{array}{l}\text { (E) Pregunta 1. Los estudiantes } \\
\text { escanean la lectura (para obtener } \\
\text { detalles). }\end{array}$ & $\begin{array}{l}\text { El 36\% de los estudiantes utilizaron el } \\
\text { escaneo para detalles (nombres, direcciones, } \\
\text { fechas) de los textos. Fueron capaces de } \\
\text { responder a las preguntas formuladas por el } \\
\text { profesor con respecto a los detalles del texto. } \\
\text { Por otro lado se observó que el } 64 \% \text { de los } \\
\text { estudiantes no utilizaban la exploración. }\end{array}$ \\
\hline & $\begin{array}{l}\text { (E) Pregunta 2. El profesor utiliza las } \\
\text { técnicas de lectura extensiva e } \\
\text { intensiva con los estudiantes }\end{array}$ & $\begin{array}{l}\text { Al revisar los tipos de lectura (intensivo y } \\
\text { extenso) el tema número seis, la profesora no } \\
\text { utilizó las técnicas de lectura extensiva e } \\
\text { intensiva con los estudiantes. Así que el } \\
100 \% \text { de los estudiantes no supo o practicó } \\
\text { este tipo de lectura en el aula y fuera de ella. }\end{array}$ \\
\hline $\begin{array}{l}\text { 2.-Identificar las técnicas } \\
\text { utilizadas por el profesor para } \\
\text { enseñar vocabulario al leer }\end{array}$ & $\begin{array}{l}\text { (P) El profesor enseña vocabulario } \\
\text { usando objetos reales, lenguaje } \\
\text { corporal, conjuntos de palabras, } \\
\text { técnica de definición, sinónimos y } \\
\text { antónimos, técnica ilustrativa o }\end{array}$ & $\begin{array}{l}\text { Según el uso de técnicas (objetos reales, } \\
\text { cuerpo, conjuntos de palabras, definición, } \\
\text { sinónimos y antónimos, ilustrativo y } \\
\text { contextualización, escalas e hipónimos) para } \\
\text { enseñar vocabulario. Los estudiantes no } \\
\text { aprendieron vocabulario con ninguna de las }\end{array}$ \\
\hline
\end{tabular}




\begin{tabular}{|c|c|c|}
\hline & $\begin{array}{l}\text { contextualización, } \\
\text { hipónimos. }\end{array}$ & $\begin{array}{l}\text { técnicas mencionadas anteriormente porque } \\
\text { la profesora no los usó en el aula. Los } \\
\text { significados de palabras desconocidas fueron } \\
\text { dados por la traducción directa. }\end{array}$ \\
\hline \multirow[t]{7}{*}{$\begin{array}{l}\text { 3.- Deducir las razones por las } \\
\text { que los estudiantes de octavo } \\
\text { año "a" de Educación Básica de } \\
\text { la } \quad \text { Unidad } \quad \text { Educativa } \\
\text { "Maldonado" carecen de } \\
\text { vocabulario al leer. }\end{array}$} & $\begin{array}{l}\text { (E) Pregunta 3. Los estudiantes tratan } \\
\text { de obtener el significado de palabras } \\
\text { desconocidas por sí mismos. }\end{array}$ & $\begin{array}{l}\text { El } 29 \% \text { de los estudiantes trataron de obtener } \\
\text { el significado de palabras desconocidas y el } \\
\text { otro } 71 \% \text { no lo hizo. Mostrando que la } \\
\text { mayoría de los estudiantes no saber cómo } \\
\text { usar un diccionario o no han traído un } \\
\text { diccionario a la clase de inglés }\end{array}$ \\
\hline & $\begin{array}{l}\text { (E) Pregunta 4. Los estudiantes } \\
\text { preguntan a la profesora por el } \\
\text { significado de alguna palabra de } \\
\text { inglés. }\end{array}$ & $\begin{array}{l}\text { El } 7 \% \text { de los estudiantes le pidió a la } \\
\text { profesora el significado de palabras } \\
\text { desconocidas. Por otro lado el } 93 \% \text { no lo } \\
\text { hizo. Se cree que los estudiantes están } \\
\text { avergonzados o temerosos de preguntarle } \\
\text { algo a la profesora. No confían en su } \\
\text { profesora }\end{array}$ \\
\hline & $\begin{array}{l}\text { (E) Pregunta 5. Los estudiantes usan } \\
\text { palabras nuevas en situaciones de la } \\
\text { vida real. }\end{array}$ & $\begin{array}{l}\text { Al discutir el vocabulario utilizado para las } \\
\text { situaciones de la vida real, el } 100 \% \text { de los } \\
\text { estudiantes no aplicó el nuevo vocabulario } \\
\text { aprendido en el aula a sus situaciones reales. } \\
\text { Estos estudiantes no pudieron usar nuevas } \\
\text { palabras aprendidas en diálogos, contando } \\
\text { historias, y puntos de vista sobre temas y } \\
\text { temas de la vida real. }\end{array}$ \\
\hline & $\begin{array}{l}\text { (E) Pregunta 6. El docente practica el } \\
\text { hábito de leer con los estudiantes. }\end{array}$ & $\begin{array}{l}\text { Se notó que el } 36 \% \text { de los estudiantes } \\
\text { practican el hábito de lectura dentro de } \\
\text { la clase, mientras que el } 64 \% \text { de ellos no } \\
\text { tienen una buena guía para leer. No seguir el } \\
\text { proceso de lectura. }\end{array}$ \\
\hline & $\begin{array}{l}\text { (E) Pregunta 7. Los estudiantes son } \\
\text { alentados por el docente a leer. }\end{array}$ & $\begin{array}{l}\text { Se observó que el } 100 \% \text { de los estudiantes no } \\
\text { son alentados por la profesora a leer para que } \\
\text { los estudiantes pierdan interés por leer en } \\
\text { inglés. }\end{array}$ \\
\hline & $\begin{array}{l}\text { (E) Pregunta } 8 . \text { Los estudiantes } \\
\text { entienden el texto. }\end{array}$ & $\begin{array}{l}\text { En la clase de } 28 \text { estudiantes, se observó que } \\
6 \text { de ellos entendían el texto porque tenían } \\
\text { conocimientos previos de vocabulario } \\
\text { mientras que los otros } 22 \text { estudiantes no lo } \\
\text { hacían. Entonces se dice que el } 79 \% \text { de los } \\
\text { estudiantes no entendían el contenido de la } \\
\text { lectura porque la profesora no introdujo } \\
\text { palabras nuevas en el texto antes de } \\
\text { comenzar a trabajar con sus estudiantes. }\end{array}$ \\
\hline & $\begin{array}{l}\text { (E) Pregunta 9. Los estudiantes } \\
\text { subrayan las palabras desconocidas. }\end{array}$ & $\begin{array}{l}\text { El } 64 \% \text { de los estudiantes subrayaron } \\
\text { palabras desconocidas mientras leían. Por } \\
\text { otra parte } 36 \% \text { de ellos no lo hicieron. No } \\
\text { estaban interesados en leer el texto. }\end{array}$ \\
\hline
\end{tabular}

Fuente: Guía de observación aplicada a la muestra Elaborado por: Cando Liliana, 2016

\section{Análisis de la pauta de la entrevista aplicada al profesor La pauta de la encuesta aplicada al profesor reveló los siguientes resultados:}


Según las pregunta 1, el profesora practica el hábito de lectura con sus estudiantes.

En la pregunta 2; se argumentó que una buena opción para motivar a sus estudiantes es proporcionarles temas interesantes, diferentes tipos de lecturas, realizar una inducción según la lectura. Los estudiantes no fueron motivados con actividades de inducción para la lectura.

En la pregunta 3; la profesora mencionó que utiliza muchos tipos de lecturas o textos con sus estudiantes como: periódicos, novelas, cuentos o libros de historia, libros, etc. Sin embargo, no se seguía una secuencia de lectura.

En la pregunta 4; la profesora siempre utiliza el escaneo con sus estudiantes cuando leían. En las observaciones, se evidenció que un porcentaje menor de los estudiantes utilizaba el raspado en la lectura. Esto causó que los estudiantes que denotaron la lectura pudieran conseguir los detalles importantes de la misma.

Según la pregunta 5, la profesora mencionó que la exploración es el tipo más apropiado de lectura para sus estudiantes, ya que se puede encontrar información importante (palabras clave, direcciones, nombres) a través de explorar el texto.

En la pregunta 6: la profesora mencionó que el inglés es importante en nuestras vidas, ya que actualmente el idioma inglés, utiliza en todas partes. Los estudiantes necesitan aprender vocabulario ya que es una fuente principal para aprender el idioma inglés. Saber inglés permite a los estudiantes conocer y descubrir diferentes personas y culturas.

En la pregunta 7, la profesora enseño vocabulario de diferentes maneras usando: tarjetas flash, fotos, cuerpo, gestos. Según las observaciones, el significado de las palabras fue dado directamente sin usar ninguna técnica para enseñar vocabulario.

De acuerdo con la pregunta 8; la profesora fue presentando diversas técnicas de enseñanza de vocabulario, señalando que conoce y utiliza objetos reales, cuerpo, gesto, frases ilustrativas. Cuando se observaron las clases no se hizo uso de ninguna de estas técnicas.

En la pregunta número 9; la profesora argumentó que todas las técnicas son buenas pero los estudiantes aprenden mejor con objetos reales, ya que les ayuda a entender las lecturas. Sin embargo, durante las observaciones no aplicaron los objetos reales para enseñar vocabulario.

Finalmente, la docente reflexionó, indicando que los estudiantes no aprenden vocabulario, porque no les gusta aprender inglés. Los estudiantes no aprenden vocabulario porque fácilmente se distraen. Hay estudiantes que nunca han sido expuestos con el idioma inglés y provienen de las parroquias rurales. 


\section{RESULTADOS}

Cuando se asistió a las clases de inglés, se observó varios inconvenientes con los estudiantes de octavo año; entre ellas, que los estudiantes tienen malos hábitos de lectura. Durante varias observaciones realizadas, los estudiantes no siguen un proceso de lectura, no son orientados con una secuencia ordenada de pasos para leer. El profesor utiliza el escaneo en todas las asignaciones de lectura.

Los estudiantes utilizaron la exploración durante el desarrollo de las tareas del libro. Este tipo de lectura es requerida para responder preguntas o llenar artículos con detalles de lectura. Escanearon la lectura más de una vez para obtener pequeños detalles y terminar sus tareas. Sin embargo, no fueron completamente exitosos, ya que no pudieron entender todo el contenido, debido a que no seguía una secuencia de lectura; como lectura rápida y elaboración de las palabras desconocidas al comienzo.

La mayoría de los estudiantes no entendían el texto cuando leían, porque la profesora no enseñaba suficiente vocabulario y los estudiantes no trataban de aprender más palabras por sí mismos.

A lo largo de la aplicación de la observación, se determinó que algunos estudiantes utilizaban el diccionario de inglés para buscar el significado de las palabras desconocidas. Pero muchos de ellos, no sabían el uso correcto del mismo y no obtuvieron el significado, presentando frustración. Se ha notado que los estudiantes subrayan palabras desconocidas pero están esperan que el profesor les indique el significado, sin embargo se omitía algunas palabras que los estudiantes no conocen.

Luego dejó un gran vacío de ignorancia de las palabras de los estudiantes. Esto también se debe a que los estudiantes no se atreven a preguntar a la profesora por vergüenza o porque sus compañeros se burlan de ellos. Esto también depende de la personalidad de cada estudiante y la confianza que los estudiantes tienen hacia su profesora.

\section{CONCLUSION}

El escaneo fue el tipo de lectura utilizada en clase, que presentó resultados deficientes en su desarrollo y se determinó que durante el proceso de lectura, la profesora no orientó a los estudiantes para mejorar sus hábitos siguiendo una secuencia lógica de pasos.

Se detectó que el profesor no aplica técnicas de lectura cuando enseñaba el vocabulario, ni tampoco se indica el significado de las palabras desconocidas por los estudiantes. Es decir, el profesor solo utiliza la traducción al español.

Los estudiantes no fueron motivados por la profesora para leer. Es decir, que los estudiantes no se sintieron confiados para pedir el significado de las palabras desconocidas. No tratan de aprender vocabulario por sí mismos; 
a veces porque no saben cómo y a veces porque algunos de ellos provienen de zonas rurales y no tienen bases de lengua inglesa.

\section{RECOMENDACIONES}

La unidad educativa "Maldonado" debería implementar un curso para profesores sobre cómo ayudar a los estudiantes a mejorar sus hábitos de lectura e implementar los tipos de lectura siguiendo una secuencia ordenada de pasos para lograr el aprendizaje requerido.

Las autoridades y los profesores de inglés deben diseñar un libro guía que contenga las técnicas, estrategias y los pasos metodológicos para la enseñanza del vocabulario. Como resultado de cursos profesionales promovidos a la institución.

Los profesores de inglés deben utilizar la información de esta investigación y su marco teórico para promover los hábitos de lectura en su unidad educativa.

\section{References:}

1. Bamberger, R. (1975). Promoting the Reading Habit. Retrieved Julio 15, 2016, from http://unesdoc.unesco.org/images/0001/000134/013412eo.pdf

2. Beale, A. M. (4 de febrero de 2014). Skimming And Scanning: Two Important Strategies For Speeding Up Your Reading. Recuperado el 2 de agosto de 2016, de http://www.howtolearn.com/2013/02/skimming-and-scanning-twoimportantstrategies-for-speeding-up-your-reading/

3. Godman, K. a. (1998). Goodmann, Y., \& Goodmann, K. (1998). Chinese Edition. Kenneth, Y. (1998).

4. Nuttall, C. (1982). Teaching reading skills in a foreign language. London.

5. Nuttall, C. (1996). Teaching Reading Skills in a foreign language.

6. S, T. (2002). How to teach vocabulary. En T. S, How to teach vocabulary.

7. Torres, M. (2010). Basic Methodology for Teaching English.

8. Wilkins, David A. (1972).Linguistics in language teaching. Edward Arnold, London. 